\title{
Nuclease-resistant synthetic ribozymes: developing a new class of therapeutics
}

\author{
Nassim Usman and Lawrence M. Blatt \\ Ribozyme Pharmaceuticals Inc., Boulder, Colorado, USA \\ Address correspondence to: Nassim Usman, Research and Development, Ribozyme Pharmaceuticals Inc., 2950 Wilderness Place, \\ Boulder, Colorado 80301, USA. Phone: (303) 546-8112; Fax: (303) 449-6995; E-mail: usmann@rpi.com.
}

The discovery of ribozymes by Cech and Altman has fundamentally changed the view of the function of RNA in chemistry, biology, and medicine (1-3). Prior to this discovery, RNA had been viewed as a passive molecule that only carried information or provided structure to RNA-protein complexes. It is now clear that RNA can act as an enzyme and is capable of catalyzing RNA splicing and cleavage, as well as several other chemical reactions. These novel activities of RNA now permit the development of enzymatic RNA molecules as therapeutic agents that can suppress the expression of deleterious proteins by catalyzing the trans-cleavage of the corresponding mRNAs (4). RNA targets for ribozyme-based therapeutics may encode oncoproteins, growth factors, proinflammatory cytokines and their corresponding cell-surface receptors, and signal transduction molecules; viral and microbial mRNAs or genomic RNAs are also readily cleaved by this approach.

RNA-cleaving ribozymes gain their target specificity from Watson-Crick base-pairing between the ribozyme's binding-arm sequences and sequences that flank the cleavage site of the target RNA. Once bound, their mechanism of cleavage involves attack of the $2^{\prime}-\mathrm{OH}$ that is $5^{\prime}$ to the scissile bond in the target, thus destabilizing the target RNA's phosphate backbone. Upon cleavage, the resultant products dissociate from the ribozyme complex and the ribozyme is released and may bind and cleave other targets again. The cleavage event renders the mRNA untranslatable and leads to further degradation of the target by cellular ribonucleases.

With the recent completion of the sequencing of the human genome and several viral and bacterial pathogens, the number of potential ribozyme drug targets is enormous. This number will increase as the functions of all the genes in the human genome become clearer and the number of pathogens being sequenced increases. However, if they are to reach the clinic, ribozymes directed at these targets will need to be designed so that they can survive in nuclease-rich biological tissues and fluids, have a favorable pharmacokinetic $(\mathrm{PK})$ profile, and prove effective in preclinical cell culture and animal models.
Several groups have demonstrated the efficacy of ribozymes in cell culture and animal models. To date, only three ribozymes - one vector-expressed and two synthetic - have been reported in clinical trials. The first, a retrovirally expressed ribozyme that targets the HIV tat and rev exons, entered clinical testing in late 1996 and is currently in phase II testing for patients with AIDS-related lymphoma. The second, ANGIOZYME, is directed against the mRNA for Flt-1 (VEGF-R1), the high-affinity receptor for VEGF. This antiangiogenic ribozyme entered phase I trials in late 1998, phase I/II trials in late 1999, and is currently in phase II trials for several tumor types. The third, HEPTAZYME, a ribozyme targeting the $5^{\prime}$-untranslated region (5'-UTR) of the hepatitis C virus (HCV) RNA genome, has recently completed a phase I/II clinical trial in patients with chronic hepatitis $\mathrm{C}$.

This perspective will focus on nuclease-resistant, chemically synthesized ribozymes, as exemplified by ANGIOZYME and HEPTAZYME. These ribozymes can be readily synthesized, can be administered subcutaneously (s.c.) or intravenously (i.v.), have excellent specificity, are well tolerated, and function well in several in vitro and in vivo model systems. The clinical efficacy of these two ribozymes is being assessed currently. If they, or others in preclinical development, show a clinical effect, then the potential utility of nuclease-resistant ribozymes in medicine will be realized, with many more applications to follow.

Ribozyme design, stabilization, and synthesis Since cellular fluids and tissues contain abundant ribonucleases, an all-RNA ribozyme would ordinarily be degraded too rapidly to function well in vivo. To protect against degradation by host nucleases, ribozymes have been developed that contain stabilizing chemical modifications, primarily at the $2^{\prime}-\mathrm{OH}$ position of the sugar residue in each nucleotide $(5,6)$. Figure 1 shows a generic, nuclease-resistant ribozyme that was identified through selective modifications to test its various structural features (5). Modifications that provide stability include the use of 2'-O-Me nucleotides in place of 2'hydroxyls throughout most of the ribozyme sequence. 2'-O-Me nucleotides are naturally occurring and thus 


\section{Nucleic acid therapeutics \\ Bruce A. Sullenger, Series Editor}

not expected to confer any toxicity on the modified ribozyme. Additionally, an inverted deoxyabasic sugar residue is placed at the $3^{\prime}$ end of the ribozyme to provide a 3'-3' linkage, and three or four phosphorothioate linkages are inserted at the $5^{\prime}$ end of the ribozyme. Although phosphorothioate linkages, which are used in greater numbers in typical DNA-based antisense molecules, can promote nonspecific binding to cellular proteins and other biomolecules (7), ribozymes containing this limited number of phosphorothioate linkages are less promiscuous in their interactions. Five purine nucleotides within the core must remain as unmodified ribonucleotides for the structure to maintain its catalytic activity. Ribozymes carrying all of these modifications are inefficiently recognized by cellular nucleases and have greatly improved biological stability. These chemically modified nuclease-resistant ribozymes are readily synthesized using a solid-phase phosphoramidite method (8), in which nucleotides are added stepwise to the growing oligonucleotide chain. This method can be highly efficient and allows for either high throughput synthesis of hundreds of ribozymes per week or the large-scale synthesis of kilogram quantities of material to be used in clinical studies.

Catalytic properties of ribozymes

For the type of nuclease-resistant ribozyme shown in Figure 1, the sole sequence requirement in the substrate RNA is a uridine (U) nucleotide followed by a C, A, or $\mathrm{U}$ (a so-called UH site). Since UH sites occur approximate- ly once every five nucleotides, numerous ribozyme cleavage sites can be identified on any target RNA. In practice, however, one must screen a large number of these sites to find one that is accessible, since many will be encumbered by the secondary or tertiary structure of the RNA target and/or bound proteins.

In addition to their versatility and their use of biologically normal chemical modifications (along with a limited number of phosphorothioate linkages) to improve stability in vivo, the principal advantages of these ribozymes is their sequence specificity. Typically, the target site is about 15 nucleotides in length, but since these ribozymes bind their cognate RNA using two independent binding arms 6-7 nucleotides in length (interrupted by the nucleotide being cleaved), ribozyme binding to the target is weaker than it would be to a contiguous sequence of 13-15 nucleotides. This weak binding leads to better sequence specificity, since it makes the ribozyme more sensitive to the effects of mismatches. Furthermore, since the target is a well-defined and unique 15nucleotide stretch of RNA, ribozymes may have an advantage over small molecule-based drugs, which typically interact not only with their target but also with all structurally related proteins.

The delivery of synthetic nuclease-resistant ribozymes into cultured cells is complicated by the need for a carrier, usually a lipid-based compound. This technical challenge is a major hurdle in the early screening of optimal ribozymes prior to preclinical in vivo

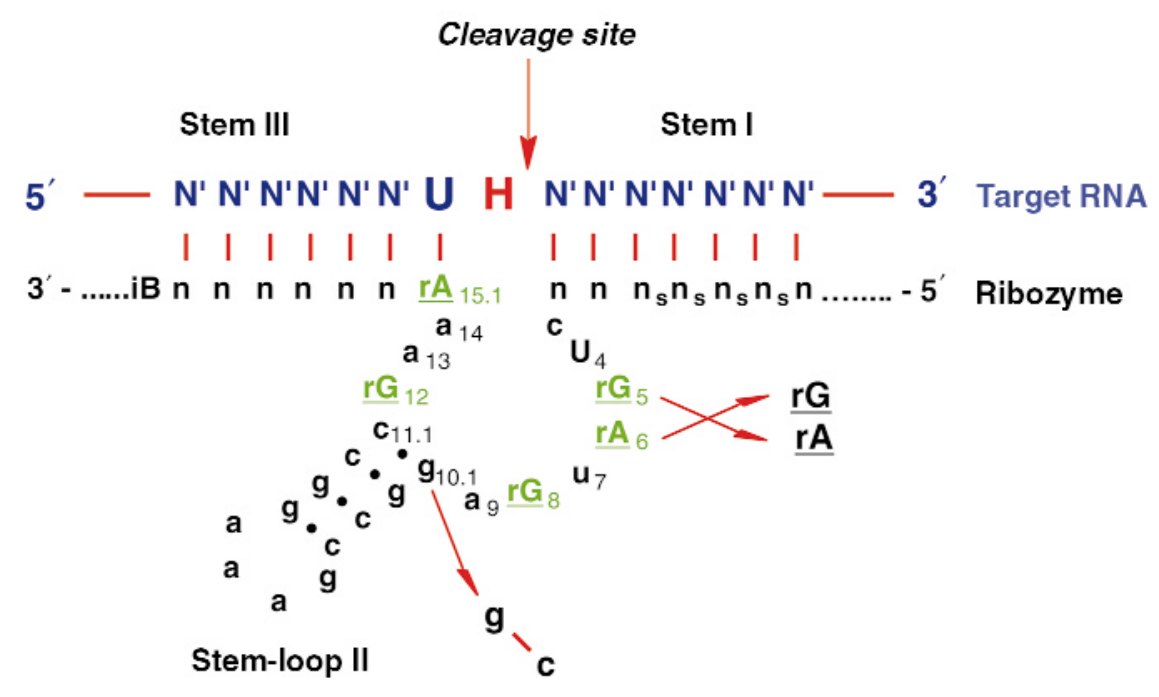

Figure 1

Structure of a general nuclease-resistant ribozyme bound to a generic target RNA. The ribozyme consists primarily of 2'-O-Me nucleotides (lower case). The $3^{\prime}$ and $5^{\prime}$ ends are protected by an inverted $3^{\prime}-3^{\prime}$ deoxyabasic sugar (iB) and four phosphorothioate linkages (s), respectively. The five purine nucleotides ( $\mathrm{rA}$ or $\mathrm{rG}$ ) in the catalytic core (shown in green) are required for catalytic activity. A single non-natural carbon containing pyrimidine ana$\log \left(2^{\prime}\right.$-C-allyluridine) at $\mathrm{U} 4$ was used to stabilize the core and maintain activity. The ribozyme binds its cognate target via Watson-Crick base-pairing. In the target strand, cleavage takes place $3^{\prime}$ to the unpaired nucleotide $\mathrm{H}(\mathrm{H}=\mathrm{A}, \mathrm{C}$, or $\mathrm{U})$. This type of ribozyme has an ex vivo serum $\mathrm{t}_{1 / 2}=10$ days. 
testing. Unexpectedly, however, no such vehicle is needed in vivo. It appears that cells in vivo have a different uptake potential, and the clinical formulations required to date have been simple saline solutions.

\section{Ribozymes in oncology}

Ribozyme-based therapeutics for cancer might be devised to block tumorigenesis itself or might act at a later stage, to inhibit tumor growth or prevent metastasis. Thus, mRNAs encoding oncogenes, growth factors or growth factor receptors, signal transduction molecules, and cell cycle proteins could all offer attractive targets. Angiogenesis, the formation of new blood vessels, is also required for sustained tumor growth (9), making the VEGF pathway another promising target for either small molecule- or nucleic acid-based therapeutics. Of particular interest are the receptor tyrosine kinases Flt-1 (VEGF-R1) and KDR (VEGF-R2), which are expressed primarily on endothelial cells and activate cell division in response to $\operatorname{VEGF}(10,11)$.

Because of the critical role of VEGF in tumor angiogenesis, we have devised ribozymes specific for Flt-1 and KDR mRNAs. The lead candidate, ANGIOZYME, targets the mRNA for Flt- 1 and is superior to anti-KDR ribozymes in its ability to inhibit tumor metastasis in a syngeneic murine model of lung cancer (12). Indeed, ANGIOZYME has now been tested in several animal models of angiogenesis, including the rat corneal pocket assay, in which VEGF-containing filter disks are implanted in the cornea to induce vascular growth. Treatment with ANGIOZYME significantly reduces this response, while its attenuated (catalytically inactive) control has no effect (13).

To investigate the potential use of ANGIOZYME against solid tumors, the ribozyme was studied in a murine model, using a highly metastatic variant of Lewis lung carcinoma (12). Continuous i.v. infusion of ANGIOZYME for 14 days following inoculation inhibits primary tumor growth in a dose-dependent manner and results in a statistically significant and dose-dependent inhibition of lung metastasis, leading to an approximately $80 \%$ reduction at the two highest doses tested (12). Pavco and colleagues (12) have also tested ANGIOZYME with a human tumor line, using a colorectal cancer xenograft model to further evaluate the antimetastatic effects of the molecule. These authors implanted a colorectal tumor cell line into the spleens of nude mice and then initiated a 28-day course of subcutaneously delivered ANGIOZYME at a range of doses. Five days after inoculation, they removed the primary tumor, and, 36 days later, they determined the animals' hepatic metastatic burden. As shown in Figure 2, higher doses of ANGIOZYME reduced the median number of liver metastases from $>100$ for the control to 5 and 0 for the 108 and $300 \mathrm{mg} / \mathrm{m}^{2}$ dose groups, respectively, demonstrating that ANGIOZYME delivered by the s.c. route has potent antimetastatic activity.

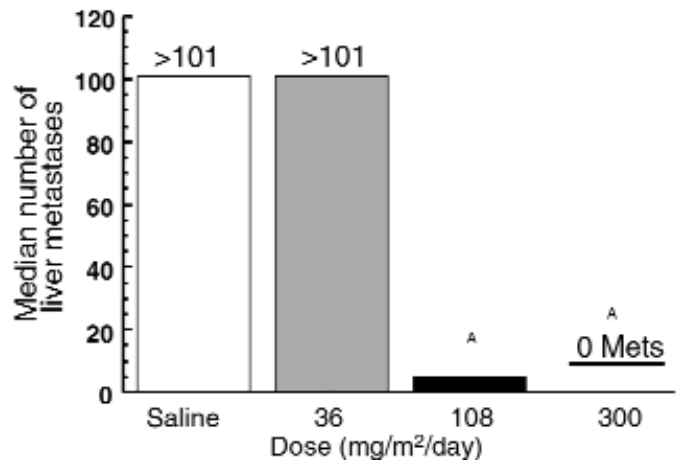

Figure 2

Inhibition of colorectal cancer metastases to the liver by ANGIOZYME. The number of metastases to the liver 41 days after intrasplenic inoculation of KM12L4a cells is shown. ANGIOZYME was administered by continuous s.c. infusion for 28 days starting on day 3 after inoculation. Details are described in ref. 12. ${ }^{A} P<0.05$.

A clinical trial, designed to test ANGIOZYME's safety, tolerability, and PK profile in healthy volunteers, began in late 1998 (14). As had been seen in preclinical studies using various animal models, the phase I study of ANGIOZYME confirmed that the agent is maintained in the plasma for several hours following s.c. or i.v. administration. ANGIOZYME administration appears to be well tolerated after a single 4-hour i.v. infusion of up to $30 \mathrm{mg} / \mathrm{m}^{2}$ or a single s.c. bolus of 20 $\mathrm{mg} / \mathrm{m}^{2}$. We continued clinical testing of ANGIOZYME in patients with a history of cancer, again using a single-dose i.v. infusion or s.c. bolus design, but at higher doses of 100 and $300 \mathrm{mg} / \mathrm{m}^{2}$. Again, ANGIOZYME was well tolerated by both routes of administration, and it maintained its plasma levels for prolonged periods, up to 24 hours when delivered by the s.c. route.

The data from the first two phase I studies and the lack of toxicity in a 28-day monkey toxicology study (discussed below) prompted us to initiate a multidose phase I/II trial for ANGIOZYME. The study was designed to demonstrate safety and determine the PK profile after repeated doses in humans and to follow biological markers related to angiogenesis. In the initial part of this study, cancer patients whose disease was refractory to approved treatments were treated using daily doses of ANGIOZYME at 10, 30, 100, and $300 \mathrm{mg} / \mathrm{m}^{2}$ s.c. (15). Following the 29-day dosing period, patients had a 7-day washout period followed by restaging. If there was no disease progression, patients were eligible to receive ANGIOZYME until progression with no interruption. Once the dose escalation was completed, a dose expansion cohort of 15 patients was enrolled at a dose of $100 \mathrm{mg} / \mathrm{m}^{2}$, based on PK results that were similar to those seen in the phase Ia and Ib studies. Enrollment of the expanded cohort was recently completed. To date, patients have been on drug for 

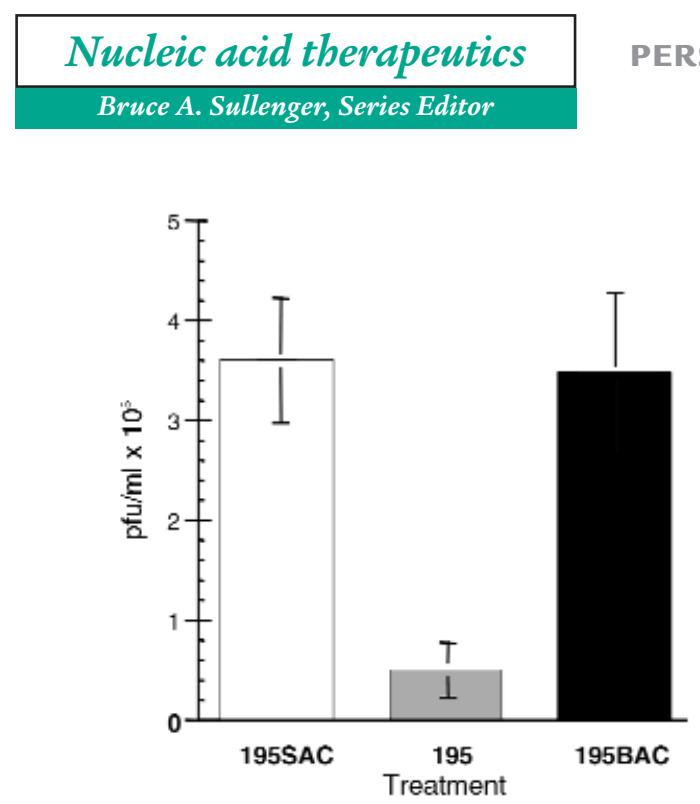

Figure 3

Efficacy and mechanism of action of an anti-HCV ribozyme. Treatment of cells infected with HCV-poliovirus, using a ribozyme (HEPTAZYME) directed at site 195 of the HCV genome, results in a significant, approximately $90 \%$ inhibition of replication $(P<0.01)$, while treatment with either a SAC or a BAC results in no inhibition of viral replication.

up to 7 months (15) and ANGIOZYME has been well tolerated by all subjects. Phase II efficacy trials in specific cancers have been initiated and results are expected in late 2001.

Ribozymes in viral diseases

Viruses are obligate cellular parasites carrying either an RNA- or a DNA-based genome. Although viral infections of any kind might be treated with ribozymes that cleave essential coding RNAs, the RNA viruses - and others whose replication strategy involves an RNA intermediate - seem particularly well suited to attack by this class of therapeutics. We have focused some of our efforts on devising ribozymes to block the replication of hepatitis $\mathrm{C}$ virus (HCV), an RNA virus that causes substantial human disease worldwide.

Chronic infection with $\mathrm{HCV}$ can lead to cirrhosis, liver failure, and hepatocellular carcinoma over a period of 20 to 30 years (16). A recent estimate by the Centers for Disease Control and Prevention places the number of chronically infected Americans at approximately 4.5 million, making HCV infection approximately four times more prevalent then HIV infection (17). Worldwide, the prevalence is similar to that found in the US.

$\mathrm{HCV}$ is a 9.5-kilobase, plus-strand RNA virus and a member of the human flavivirus family (18). Although the sequence of $\mathrm{HCV}$ varies among clinical isolates, the 5 '-UTR of the genome is highly conserved (19). This RNA sequence contains an internal ribosome entry site that allows for translation initiation without the need for a $5^{\prime}$-cap structure (20).

To investigate the potential of nuclease-resistant ribozymes to treat chronic hepatitis $\mathrm{C}$, we scanned a sequence alignment of about $50 \mathrm{HCV}$ isolates from several genotypes to find regions of $100 \%$ conservation in the $5^{\prime}$-UTR that might be suitable for ribozyme cleavage. We identified 22 such sites, and, using an $\mathrm{HCV} /$ poliovirus chimera $(21,22)$ that is better suited than native $\mathrm{HCV}$ for propagation in culture, we tested several of the corresponding ribozymes for their ability to inhibit viral replication. Seven of these candidates, when added to cultures of infected cells, resulted in $50-90 \%$ inhibition of viral replication, and further analysis identified one ribozyme, targeting site 195 in the HCV genome, as a lead candidate (21).

We synthesized two control ribozymes to test the specificity of the antiviral effects of this candidate, now called HEPTAZYME. One, called a binding arm attenuated control (BAC), is closely related to HEPTAZYME but contains a sequence change in its catalytic core so that it can bind the target RNA but cannot cleave it. The other, a scrambled arm attenuated control (SAC), is similar to the BAC, but its binding-arm sequences are scrambled to prevent target binding. As shown in Figure 3 , only HEPTAZYME inhibits the $\mathrm{HCV}$ /poliovirus replication, confirming that its effects depend on a ribozyme mechanism. To test for nonspecific effects of HEPTAZYME against RNA sequences contained in the poliovirus coding region, we treated wild-type poliovirus with either HEPTAZYME, the BAC, or the SAC. As expected (and as previously reported [ref. 21]), no oligonucleotide in this experiment inhibited replication of the wild-type poliovirus. We have also found that HEPTAZYME and type 1 interferon in combination have a more potent antiviral activity than either compound individually. These experiments help to validate the strategy of using ribozymes to treat viral infections and indicate the promise of HEPTAZYME in treating chronic hepatitis C. A phase II clinical trial beginning in 2001 will ascertain the full potential therapeutic benefit of HEPTAZYME for the treatment for chronic hepatitis $\mathrm{C}$.

\section{Ribozyme toxicology and pharmacokinetics}

Two classes of toxicity must be considered in testing any novel therapeutic agent: specific toxicity, which results from the agent's interactions with its intended

\section{Table 1}

Summary of ANGIOZYME toxicology

\begin{tabular}{lcc}
\hline Study & Highest dose & Adverse effects \\
Mouse single i.v. & $3500 \mathrm{mg} / \mathrm{m}^{2}$ & None \\
Mouse 14-day i.v. & $900 \mathrm{mg} / \mathrm{m}^{2}$ & None \\
Mouse single i.v. & $900 \mathrm{mg} / \mathrm{m}^{2}$ & None \\
Monkey single i.v. and s.c. & $1200 \mathrm{mg} / \mathrm{m}^{2}$ & None \\
Mouse micronucleus i.v. & $6000 \mathrm{mg} / \mathrm{m}^{2}$ & Negative \\
Mouse 28-day s.c. & $300 \mathrm{mg} / \mathrm{m}^{2}$ & None \\
Monkey 28-day s.c. & $300 \mathrm{mg} / \mathrm{m}^{2}$ & None \\
\hline
\end{tabular}


target molecule, and nonspecific toxicity, which results from other interactions. Because nuclease-resistant ribozymes contain several naturally occurring modified nucleotides, nonspecific interactions from the modified nucleotides were not expected. Indeed, toxicology studies on ANGIOZYME (Table 1) showed no evidence of clinically significant adverse events, even for doses in excess of the highest doses used in human clinical trials, $300 \mathrm{mg} / \mathrm{m}^{2}$. The typical dose required for efficacy ranges from 30 to $100 \mathrm{mg} / \mathrm{m}^{2}$. Hence, the downregulation of Flt- 1 does not appear to cause any specific toxicity in the systems tested (23). Among the minor effects noted in a 28-day monkey study, kidney and liver cells accumulated basophilic granules, probably as a result of drug accumulation in these tissues. These animals showed no abnormal clinical chemistry, however, and the basophilic granules resolved after the treatment ended. There were some mild injection site reactions that also resolved in the recovery group. The preclinical toxicological profile of HEPTAZYME was similar to that of ANGIOZYME.

Overall, this novel class of molecules seems to be well tolerated in all studies to date. However, in addition to satisfying safety concerns, an agent must demonstrate satisfactory PK and tissue distribution and must enter target cells and function efficiently in vivo. Pharmacodynamic (PD) and PK studies in animals (24) confirm that nuclease-resistant ribozymes are taken up efficiently into cells in vivo. Data from these studies are also useful because they can be used, in conjunction with PK parameters obtained in humans, to define the doses of ribozymes required to exert a PD effect in human subjects following administration by any of several routes.

Sandberg et al. (ref. 25 and unpublished data) studied the PK behavior of ${ }^{32} \mathrm{P}$-labeled ANGIOZYME after systemic i.v., s.c., or intraperitoneal (i.p.) administration in both murine and primate systems. Regardless of route, ANGIOZYME was detected in all tissues examined, with the highest concentrations found in kidney, liver, and femur. A comparison of plasma and tumor tissue concentrations of ANGIOZYME following administration of a $100 \mathrm{mg} / \mathrm{m}^{2}$ dose indicate that both the s.c. and i.p. routes gave extended plasma residence times compared with the i.v. route. Furthermore, ANGIOZYME can be detected in tumor tissue of mice harboring Lewis lung carcinomas for up to 72 hours after a single i.v. bolus administration, indicating that the ribozyme can be found in some tissues far beyond its plasma residence time. Similar studies using larger species, the rat and monkey, confirm that s.c. administration leads to good bioavailability, suggesting that this more convenient route of administration may be effective and practical in patients, as has indeed been borne out in clinical trials with ANGIOZYME $(14,15)$. Once-daily s.c. bolus dosing appears to be clinically feasible and is being used in the ongoing efficacy trials for both ANGIOZYME and HEPTAZYME.
Finally, we have used a fluorescently labeled analogue of ANGIOZYME to define its tissue distribution in target organs. The appearance of fluorescent ribozyme in tissue sections from treated animals is consistent with the evidence of biological activity by the ribozyme. For example, in the model of liver metastasis described above, fluorescence can be seen in the liver 30 minutes to 48 hours after dosing, and $50-75 \%$ of hepatocyte nuclei stain brightly at 30 minutes after s.c. injection, with the remaining nuclei also moderately positive (our unpublished data). Nuclear fluorescence persists at faint to moderate levels even as late as 48 hours after injection.

\section{Conclusions}

Nuclease-resistant synthetic ribozymes are now being developed for a number of therapeutically important targets. For example, the antiangiogenic ribozyme ANGIOZYME, which targets the VEGF receptor Flt-1, has demonstrated potent activity in several preclinical oncology models, has been well tolerated in both toxicology and phase I/II clinical studies, and has produced a favorable PK profile. A similar synthetic ribozyme, HEPTAZYME, targeting a conserved region of the HCV RNA, has potent antiviral activity in cell culture and is currently being studied in patients with chronic hepatitis C. In theory, nuclease-resistant ribozymes can be designed to any target with only the knowledge of the target RNA sequence. The recently completed sequencing of the human genome and of the genomes of several pathogenic species provides a large number of potential ribozyme targets that may be of benefit in clinical medicine. These ribozymes can be stabilized to prevent biological degradation using a variety of chemical modifications, the majority of which are naturally occurring. Thus, nuclease-resistant synthetic ribozymes are emerging as a new and broadly useful class of therapeutic agent.

\footnotetext{
1. Cech, T.R., Zaug, A.J., and Grabowski, P.J. 1981. In vitro splicing of the ribosomal RNA precursor of Tetrabymena: involvement of a guanosine nucleotide in the excision of the intervening sequence. Cell. 27:487-496.

2. Guerrier-Takada, C., Gardiner, K., Marsh, T., Pace, N., and Altman, S. 1983. The RNA moiety of ribonuclease $P$ is the catalytic subunit of the enzyme. Cell. 35:849-857.

3. Cech, T.R. 1992. Ribozyme engineering. Curr. Opin. Struct. Biol. 2:605-609.

4. Usman, N., and Stinchcomb, D.T. 1996. Design, synthesis and function of therapeutic hammerhead ribozymes. Nucleic Acids and Molecular Biology. 10:243-264.

5. Beigelman, L., et al. 1995. Chemical modification of hammerhead ribozymes: catalytic activity and nuclease resistance. J. Biol. Chem. 270:25702-25708.

6. Usman, N., and McSwiggen, J. 1995. Catalytic RNA (ribozymes) as drugs. In Annual reports in medicinal chemistry. Academic Press Inc. San Diego, California, USA. 285-294.

7. Henry, S.P., Monteith, D., and Levin, A.A. 1997. Antisense oligonucleotide inhibitors for the treatment of cancer. II. Toxicological properties of phosphorothioate oligodeoxynucleotides. Anticancer Drug Des. 12:395-408.

8. Wincott, F.E., et al. 1995. Synthesis, deprotection, analysis and purification of RNA and ribozymes. Nucleic Acids Res. 23:2677-2684.

9. Folkman, J. 1971. Tumor angiogenesis: therapeutic implications. N. Engl. J. Med. 285:1182-1186.

10. Thomas, K.A. 1996. Vascular endothelial growth factor, a potent and selective angiogenic agent. J. Biol. Chem. 271:603-606.

11. Ferrara, N., Heinsohn, H., Walder, C.E., Bunting, S., and Thomas, G.R.
} 
1995. The regulation of blood vessel growth by vascular endothelial growth factor. Ann. NY Acad. Sci. 752:246-255.

12. Pavco, P.A., et al. 2000. Antitumor and antimetastatic activity of ribozymes targeting the messenger RNA of vascular endothelial growth factor receptors. Clin. Cancer Res. 6:2094-2103.

13. Parry, T.J., et al. 1999. Bioactivity of antiangiogenic ribozymes targeting Flt-1 and KDR mRNA. Nucleic Acids Res. 27:2569-2577.

14. Sandberg, J.A., et al. 2000. Pharmacokinetics and tolerability of an antiangiogenic ribozyme (ANGIOZYME) in healthy volunteers. J. Clin. Pharmacol. In press.

15. Weng, D., et al. 2000. A phase I/II study of repetitive dose Angiozyme ${ }^{\mathrm{TM}}$, a ribozyme targeting the Flt-1 receptor for VEGF. The 11th NCI-EORTCAACR Symposium on New Drugs in Cancer Therapy. National Cancer Institute-European Organisation for Research and Treatment of CancerAmerican Association for Cancer Research. November 7-10, 2000. Free University, Amsterdam, The Netherlands. (Abstr.)

16. Sherlock, S. 1992. Viral hepatitis. Lancet. 339:802.

17. Hoofnagle, J. 1997. Hepatitis C: the clinical spectrum of disease. Hepatology. 26:15S-20S.

18. Choo, Q.L., et al. 1989. Isolation of a cDNA clone derived from a bloodborne non-A, non-B viral hepatitis genome. Science. 244:359-362.

19. Bukh,J., Purcell, R.H., and Miller, R.H. 1992. Sequence analysis of the 5 ' non- coding region of hepatitis C virus. Proc. Natl. Acad. Sci. USA. 89:4942-4946. 20. Wang, C., Sarnow, P., and Siddiqui, A. 1993. Translation of human hepatitis $C$ virus RNA in cultured cells is mediated by an internal ribosomebinding mechanism. J. Virol. 67:3338-3344.

21. Macejak, D.G., et al. 2000. Inhibition of hepatitus C virus (HCV)-RNAdependent translation and replication of a chimeric HCV poliovirus using synthetic stabilized ribozymes. Hepatology. 31:769-776.

22. Lu, H.-H., and Wimmer, E. 1996. Poliovirus chimeras replicating under the translational control of genetic elements of hepatitis $C$ virus reveal unusual properties of the internal ribosomal entry site of hepatitis C virus. Proc. Natl. Acad. Sci. USA. 93:1412-1417.

23. Sandberg, J.A., et al. 2000. Acute toxicology and pharmacokinetic assessment of a ribozyme (ANGIOZYME) targeting vascular endothelial growth factor receptor mRNA in the cynomolgus monkey. Antisense Nucleic Acid Drug Dev. 10:153-162.

24. Parry, T.J., Pavco, P.A., and Sandberg, J.A. 1999. Ribozyme pharmacokinetic screening for predicting pharmacodynamic dosing regimes. In Intracellular ribozyme applications: principles and protocols. J.J. Rossi and L.A. Couture, editors. Horizon Scientific Press. Wymondham, United Kingdom. 231-241.

25. Sandberg, J.A., et al. 1999. Pharmacokinetics of an antiangiogenic ribozyme (ANGIOZYME) in the mouse. Antisense Nucleic Acid Drug Dev. 9:271-277. 